\title{
Clustering Vessel Trajectories with Alignment Kernels under Trajectory Compression
}

\author{
Gerben de Vries and Maarten van Someren \\ Informatics Institute, University of Amsterdam, Sciencepark 107, 1098 XG \\ Amsterdam, The Netherlands \\ \{G.K.D.deVries,M.W.vanSomeren\}@uva.nl
}

\begin{abstract}
In this paper we apply a selection of alignment measures, such as dynamic time warping and edit distance, to the problem of clustering vessel trajectories. Vessel trajectories are an example of moving object trajectories, which have recently become an important research topic. The alignment measures are defined as kernels and are used in the kernel k-means clustering algorithm. We investigate the performance of these alignment kernels in combination with a trajectory compression method. Experiments on a gold standard dataset indicate that compression has a positive effect on clustering performance for a number of alignment measures. Also, soft-max kernels, based on summing all alignments, perform worse than classic kernels, based on taking the score of the best alignment.
\end{abstract}

Keywords: alignment kernels, trajectory compression, trajectory clustering.

\section{Introduction}

Largely due to the ubiquity of GPS receivers, moving object trajectories have become an important research topic. To gain insight into the behavior of moving objects, such as vessels, it can be useful to cluster their trajectories into groups of similar movement patterns. Essentially, moving object trajectories are a kind of multivariate time-series. Furthermore, they have the property that they are usually different in temporal length, distance traveled and the number of data points. Alignment methods, such as dynamic time warping or edit distance, are designed to handle these kinds of variations. Thus, such methods can make a suitable similarity measure for clustering moving object trajectories.

We cannot use these similarities directly in the standard, and very popular, $\mathrm{k}$-means clustering method, because this method requires an explicitly defined feature space. Kernel k-means [17] is a more recent k-means based clustering algorithms that deals with similarities directly, provided they are defined as kernels. Defining these alignments as kernels also has the advantage that they can potentially be used in other kernel based machine learning settings.

Computing alignment kernels can be expensive, because of the number of elements in a trajectory. However, vessel trajectories have the property that 
they are very regular. Without losing much information they can be compressed very well with trajectory compression techniques such as [10]. Computing an alignment between two compressed trajectories can be a lot cheaper, because of the reduction in data points. But, the quality of the alignment does not necessarily have to be the same. Compression loses information and may have a bad effect on the alignment.

In this paper we define different alignment kernels for moving object trajectories. The kernels are based on a number of well-known alignment measures. We consider two kernel versions of these measures, a classic and a soft-max one. Not all of these kernels are proper kernels, since they are not positive semi-definite. However, for simplicity, we refer to all of them as kernels. Furthermore, all kernels can be used with the kernel k-means clustering algorithm that we use in our experiment. The goal of this experiment is to investigate the performance of the different defined kernels on the task of clustering vessel trajectories. Kernels are computed on uncompressed and compressed trajectories to discover the influence of trajectory compression on this task. A number of the alignment kernels show better performance when the trajectories are compressed first.

The rest of the paper is organized as follows. In Sect. 2 we discuss related work in the fields of trajectory clustering and kernel methods for time-series. Section 3 contains the technical definitions: three kinds of alignments, two kinds of kernel definitions based on these alignments, kernel k-means clustering and trajectory compression. Our experiment and the results of clustering a hand-labeled set of vessel trajectories is described in Sect. 4. We end with some conclusions and directions for future work.

\section{Related Work}

We have not seen work on clustering moving object trajectories using an alignment kernel based approach. A number of studies address clustering time series data, see [14 for an overview. More specifically, there are a number of papers researching the problem of clustering trajectories, mostly coming from the Moving Object Database community 81315 16 19]. None of these studies consider the influence of compression on the clustering task. Also, these papers are not comparative studies between similarity measures. The authors of [19] take an alignment based approach, as we do in this paper, but use a density based clustering algorithm. Also, [15] takes a density based approach but computes the distance between trajectories based on the area between them. In [13, compression based on the minimum description length principle is applied to trajectories first and then density based clustering is used to discover sub-trajectories. Trajectories are first converted into a grid based representation and then clustered using fuzzy c-means in [16. A route similarity function very similar to dynamic time warping is described in [1. Very different is the somewhat older [8, in which the authors use a mixture of regression models based approach. Most of the above papers use a density based clustering algorithm, which assumes that clusters are not (densely) connected. However, we do not know whether this is 
actually the case when using alignment based similarities, therefore, we prefer kernel k-means.

There is also a body of work on using alignment kernels for time-series, for instance 5191112 . This work is mostly in the context of classification of handwriting data using support vector machines. Both [11] and [12] use an alignment kernel based on Dynamic Time Warping (DTW). However, 12 uses the softmax version defined in 5], whereas [1] uses a more classic version of DTW. In the following we will consider both types.

\section{$3 \quad$ Trajectory Alignment Kernels}

In this section we first define the notion of a moving object trajectory. Then we look at alignments and define different kernels for them. We also briefly review the kernel k-means clustering algorithm. Finally, we give the trajectory compression algorithm that we have used.

\subsection{Trajectories}

A moving object trajectory is defined in Definition 1 below. Note that trajectories include time.

Definition 1. A moving object trajectory in 2-dimensional space is represented by a sequence of vectors: $T=\left\langle x_{1}, y_{1}, t_{1}\right\rangle, \ldots,\left\langle x_{n}, y_{n}, t_{n}\right\rangle$. Where $x_{i}$ and $y_{i}$ represent the position of the object at time $t_{i}$. The length of a trajectory, i.e. the number of vectors, is denoted as: $|T|$. Furthermore, let $T_{i}=\left\langle x_{i}, y_{i}, t_{i}\right\rangle$.

The sample rate of trajectories is not fixed, thus the difference between consecutive values $t_{i}, t_{i+1}$ is not the same. Also, there are more dimensions to trajectories that can be derived from the $x, y, t$ information that we do not consider, such as speed and direction. In some tasks and applications these attributes might be more relevant than the absolute position $x, y$ and time $t$. In principle these dimensions could just be added to the $\langle x, y, t\rangle$ vector, but we have not considered this for the clustering task that we will define in Sect. 4. In the following we refer to a vector $\left\langle x_{i}, y_{i}, t_{i}\right\rangle$ as trajectory element or point.

\subsection{Alignments}

We define three types of alignments between two trajectories. Based on these alignment we define kernels that express similarity between trajectories in Sect. 3.3. We start with the simplest alignment, which can be considered to be the baseline alignment. The goal of an alignment is to bring the elements of two sequences, e.g. trajectories, in a correspondence, typically with the aim to maximize (or minimize) a scoring function defined on this correspondence.

Assume in the following that there is a function $\operatorname{sub}\left(S_{i}, T_{j}\right)$ that gives a score to substituting the trajectory element $S_{i}$ with $T_{j}$. This score represents the similarity between these two elements. To enhance readability, we will not make 
use of superscripts to indicate the specific type of alignment when this cannot lead to confusion.

We define different alignments for two trajectories below, following the notation and definitions from [18].

Definition 2. An alignment $\pi$, possibly with gaps, of $p \geq 0$ positions between two trajectories $S$ and $T$ is a pair of p-tuples:

$$
\pi=\left(\left(\pi_{1}(1), \ldots, \pi_{1}(p)\right),\left(\pi_{2}(1), \ldots, \pi_{2}(p)\right)\right) \in \mathbb{N}^{2 p} .
$$

The idea behind this definition is that the alignment encodes $p$ elements in each trajectory that are aligned to each other. That is, the $\pi_{1}(i)$ th element in $S$ is aligned to the $\pi_{2}(i)$ th element in $T$. Multiple elements of $S$ can be aligned to one element in $T$ and vice versa. Furthermore, not all elements have to be aligned to an element in the other trajectory, in this case an element is a gap.

Shortest Sequence Alignment. One of the simplest alignments defined on two sequences with discrete elements is the Longest Common SubSequence (LCSS) measure. It is defined as the length of the longest sequence of elements existing in both sequences, whereby gaps are allowed. Definition 3 differs from other definitions [9 19] of LCSS for sequences with continuous elements, i.e. elements with continuous values, because we will not use a threshold in our substitution function. On first glance the resulting measure does not have much in common with LCSS, thus to avoid confusion we redub it: Shortest Sequence Alignment (SSA).

Definition 3. An SSA alignment $\pi^{\mathrm{SSA}}$ is an alignment according to Definition 2. with the additional constraints that:

$$
p=\min (|S|,|T|)
$$

and

$$
\begin{aligned}
& 1 \leq \pi_{1}(1)<\pi_{1}(2)<\ldots<\pi_{1}(p) \leq|S| \\
& 1 \leq \pi_{2}(1)<\pi_{2}(2)<\ldots<\pi_{2}(p) \leq|T|
\end{aligned}
$$

Intuitively this means that all elements of the shortest sequence are aligned with different unique elements in the other sequence, hence Shortest Sequence Alignment.

Dynamic Time Warping. A very popular alignment method more specifically designed for time-series is Dynamic Time Warping (DTW). In Definition 4 we follow [5].

Definition 4. A DTW alignment $\pi^{\mathrm{DTW}}$ is an alignment according to Definition 2. but has the additional constraints that there are unitary increments and no simultaneous repetitions, thus $\forall(1 \leq i \leq p-1)$,

$$
\pi_{1}(i+1) \leq \pi_{1}(i)+1, \pi_{2}(j+1) \leq \pi_{2}(j)+1
$$


and

$$
\left(\pi_{1}(i+1)-\pi_{1}(i)\right)+\left(\pi_{2}(i+1)-\pi_{2}(i)\right) \geq 1
$$

Furthermore,

$$
\begin{aligned}
& 1=\pi_{1}(1) \leq \pi_{1}(2) \leq \ldots \leq \pi_{1}(p)=|S| \\
& 1=\pi_{2}(1) \leq \pi_{2}(2) \leq \ldots \leq \pi_{2}(p)=|T|
\end{aligned}
$$

This means that all elements in both trajectories are aligned, which might require repeating elements from a trajectory, but in the alignment we cannot simultaneously repeat an element in both trajectories. Furthermore, the start and end of trajectories are aligned by default.

Edit Distance. Edit distances are a popular method for comparing similarity of strings. This similarity is computed in terms of the number of substitutions, deletions and insertions that are required to transform one string into another string. We define (Definition [5] and [6) a version for continuous elements similar to how 4 defines it for time-series. However we chose to consider fixed gap penalties, i.e. deletion and insertion costs, because this seems more natural in the trajectory case 1

Definition 5. An edit distance alignment $\pi^{\mathrm{ED}}$ is an alignment according to Definition [2, but also has the constraints:

$$
\begin{aligned}
& 1 \leq \pi_{1}(1)<\pi_{1}(2)<\ldots<\pi_{1}(p) \leq|S| \\
& 1 \leq \pi_{2}(1)<\pi_{2}(2)<\ldots<\pi_{2}(p) \leq|T|
\end{aligned}
$$

This means that not all elements have to be aligned and there is no repetition of elements.

We define a score function for the three types of alignments below in Definition 6. Note that the function is the same for DTW and SSA alignments.

Definition 6. The score for an alignment $\pi$ of type $\phi$ of two trajectories $S$ and $T$ is equal to:

$$
s^{\phi}(\pi)=\left(\sum_{i=1}^{|\pi|} \operatorname{sub}\left(S_{\pi_{1}(i)}, T_{\pi_{2}(i)}\right)\right)+g(|S|-|\pi|)+g(|T|-|\pi|),
$$

where $g=0$ if $\phi=\mathrm{SSA}$, DTW and $g<0$ if $\phi=\mathrm{ED}$.

${ }^{1}$ In [4] the gap penalties are essentially the value of the gapped element minus a fixed constant. For trajectories this would mean that something on the edge of the map would have a higher penalty than in the middle, which we think is counter-intuitive. 
Considering the three types of alignments that are defined above, we note that the most important difference is in how they treat gaps. In the SSA case gaps get no penalty, in the DTW case gaps are treated by repeating a trajectory element, and thus get a score according to the substitution function sub, and in the edit distance case gaps have a fixed penalty $g$.

\subsection{Alignment Kernels}

Below we will give two different ways to create a kernel to express similarity between trajectories, using the alignments defined above. The first type of kernel is based on taking the score of the alignment that maximizes the score function. This differs from the second kernel which takes the soft-max of the scores of all alignments.

First, we define the substitution function to be the negative of the $L^{2}$ norm, i.e. the regular Euclidean distance, in Definition 7, Other functions are possible here, but we have not experimented with this. Note that $x$ and $y$ are of the same dimension, but $t$ is not directly comparable to $x$ and $y$, hence in the experiments we apply a weight to $t$ to make it comparable. In practice, this weight will depend on the domain and goal of one's application.

\section{Definition 7.}

$$
\operatorname{sub}\left(\left\langle x_{i}, y_{i}, t_{i}\right\rangle,\left\langle x_{j}, y_{j}, t_{j}\right\rangle\right)=-\left\|\left\langle x_{i}-x_{j}, y_{i}-y_{j}, t_{i}-t_{j}\right\rangle\right\| .
$$

Above, in Sect. 3.2, we have defined a number of alignments between two trajectories and their score functions. However, this does not give a similarity between two trajectories yet, because there are a lot of possible alignments, and corresponding scores, for one type of alignment.

One option for similarity between two trajectories is to take the score of the alignment that maximizes the respective score function $s$. We will call this similarity $\mathrm{Sim}_{\max }$ and define it below in Definition 8 , This similarity corresponds to the typical way that DTW and edit distance are defined.

Definition 8. Given two trajectories $S$ and $T$, let $\Pi_{S, T}^{\phi}$ be the set of all possible alignments between these trajectories under a certain alignment measure $\phi$, then

$$
\operatorname{Sim}_{\max }^{\phi}(S, T)=\max _{\pi \in \Pi_{S, T}^{\phi}} s^{\phi}(\pi) .
$$

However, it has been argued [518] that taking the soft-max 2 of the scores of all possible alignments leads to better kernels, because all scores are taken into account. We will call this alignment score $\operatorname{Sim}_{\text {sum }}$, because the sum over all possible alignments is taken. It is given in Definition 9 below.

Definition 9. Given two trajectories $S$ and $T$, let $\Pi_{S, T}^{\phi}$ be the set of all possible alignments between these trajectories under a certain alignment measure $\phi$, then

$$
\operatorname{Sim}_{\mathrm{sum}}^{\phi}(S, T)=\sum_{\pi \in \Pi_{S, T}^{\phi}} \exp \left(s^{\phi}(\pi)\right) .
$$

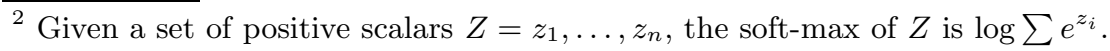


These similarities are not kernels yet. We define a kernel based on Sim $_{\max }$ in Definition 10.

Definition 10. For all trajectories $T^{i}$ and $T^{j}$ in a set of trajectories $\mathcal{T}$, we compute the $K_{\max }^{\phi}$-kernel for an alignment measure $\phi$ as:

$$
K_{\max }^{\phi}(i, j)=\operatorname{Sim}_{\max }^{\phi}\left(T^{i}, T^{j}\right),
$$

furthermore we normalize and make a kernel out of $K_{\max }^{\phi}$ by:

$$
K_{\max }^{\phi}=1-\frac{K_{\max }^{\phi}}{\min \left(K_{\max }^{\phi}\right)} .
$$

The kernel based on $\operatorname{Sim}_{\text {sum }}$ is given in Definition 11.

Definition 11. For all trajectories $T^{i}$ and $T^{j}$ in a set of trajectories $\mathcal{T}$, we compute, and normalize, the $K_{\mathrm{sum}}^{\phi}$-kernel for an alignment measure $\phi$ as:

$$
K_{\mathrm{sum}}^{\phi}(i, j)=\frac{\operatorname{Sim}_{\mathrm{sum}}^{\phi}\left(T^{i}, T^{j}\right)}{\sqrt{\operatorname{Sim}_{\mathrm{sum}}^{\phi}\left(T^{i}, T^{i}\right) \operatorname{Sim}_{\mathrm{sum}}^{\phi}\left(T^{j}, T^{j}\right)}} .
$$

The $K_{\max }$ kernels are not proper kernels in the sense that they are not Positive Semi-Definite (PSD). However it has been observed that non-PSD kernels can still work well in practice (for DTW for instance in 11]). We notice this in our experiment in Sect. 4 as well. The soft-max version of the DTW alignment kernel was proven to be PSD in [5] given a proper substitution function (e.g. the one we have defined). Furthermore, we conjecture that similar proofs are possible for the other two soft-max kernels we define, because they are very similar to either DTW or Smith-Waterman, for which there is a proof in 18 .

The authors of [5]18] also notice that the soft-max type of kernel often suffers from the diagonal dominance issue and they remedy this by taking the logarithm of this kernel. We have experimented with taking the logarithm, but this did not improve results.

The classic alignment similarities, Sim $_{\max }$, are efficiently computable via a dynamic programming approach. By replacing the max-sum algebra with a sumproduct algebra we can do the same for the Sim $_{\text {sum }}$ versions. This is shown for DTW in [5]. We can see that this works when we, for instance, work out $\operatorname{Sim}_{\mathrm{sum}}^{\mathrm{DTW}}$ as in (11).

$$
\begin{aligned}
\operatorname{Sim}_{\mathrm{sum}}^{\mathrm{DTw}}(S, T) & =\sum_{\pi \in \Pi_{S, T}} \exp \left(\sum_{i=1}^{|\pi|} \operatorname{sub}\left(S_{\pi_{1}(i)}, T_{\pi_{2}(i)}\right)\right) \\
& =\sum_{\pi \in \Pi_{S, T}} \prod_{i=1}^{|\pi|} \exp \left(\operatorname{sub}\left(S_{\pi_{1}(i)}, T_{\pi_{2}(i)}\right)\right) .
\end{aligned}
$$

To sum up, we have now defined a number of kernel functions. Two for the SSA measure: $K_{\max }^{\mathrm{SSA}}$ and $K_{\mathrm{sum}}^{\mathrm{SSA}}$. Two for the DTW measure: $K_{\max }^{\mathrm{DTW}}$ and $K_{\mathrm{sum}}^{\mathrm{DTW}}$. And a family of kernels for the edit distance measure, since these are parameterized by the value of the gapping penalty $g$ : $K_{\max }^{\mathrm{ED}}$ and $K_{\text {sum }}^{\mathrm{ED}}$. 


\subsection{Kernel K-Means}

Because the focus of the paper is on alignments, we use the relatively simple kernel k-means algorithm [17. More advanced kernel based algorithms exist, such as weighted kernel k-means [6] and support vector clustering [2].

The kernel version of k-means works in exactly the same way as regular kmeans. This means that we start out with a random initialization of $k$ clusters and at each iteration try to minimize the distance between the cluster centers and objects in the clusters until we arrive at a stable clustering (Definition 12).

Definition 12. A clustering $C$ of a set of objects $\mathcal{O}$ into $k$ partitions is:

$$
C=\left\{c_{1}, \ldots, c_{k}\right\},
$$

such that for all $o \in \mathcal{O}$, o is exactly in one $c_{i}$, where $c_{i} \subseteq \mathcal{O}$.

The difference lies in how the distance from an object to a cluster center is calculated. This is done using the assumption that a kernel represents a dotproduct in a higher dimensional feature space. Without explicitly knowing this feature space, the distance from an object to a cluster center can be calculated with these dot-products, as in Definition 13 .

Definition 13. Let $K$ be a kernel computed for a set of objects $\mathcal{O}$ and $C$ a clustering of $\mathcal{O}$. The distance from an object $o_{i} \in \mathcal{O}$ to the center of a cluster $c \in C$ is computed by:

$$
K(i, i)-\frac{2}{|c|} \sum_{o_{j} \in c} K(i, j)+\frac{1}{|c|^{2}} \sum_{o_{j}, o_{k} \in c} K(j, k) .
$$

Because different random initializations can lead to different stable partitions $C$, the kernel k-means clustering is run a number of times. The partitioning with the lowest intra cluster spread is kept as the final clustering.

\subsection{Trajectory Compression}

For trajectory compression we use the Piecewise Linear Segmentation (PLS) method proposed in [10, which is an advanced version of a classic line simplification algorithm [7. The algorithm, given in Algorithm [1, compresses a trajectory $T$ by finding the point $\left\langle x_{i}, y_{i}, t_{i}\right\rangle$ that has the largest error $\mathbf{E}_{\mu}$, as defined in Definition 14. If this error is larger than a given threshold $\epsilon$ then we keep this point and recursively apply the same procedure on the two sub-trajectories created by splitting $T$ at that point. Thus the result of applying trajectory compression to a trajectory $T$ is that the compressed trajectory $T^{C}$ is a subset of the points that are in $T$, always including the start and end points. This subset contains the most salient points of the trajectory. Thus, contrary to smoothing or resampling, existing points are not altered and no new points are created.

In Definition 14, the error $\mathbf{E}_{\mu}$ is defined as the Euclidean distance between a point $\left\langle x_{i}, y_{i}, t_{i}\right\rangle$ and the closest point on the line $\left\langle x_{1}, y_{1}, t_{1}\right\rangle,\left\langle x_{n}, y_{n}, t_{n}\right\rangle$, where the influence of the temporal dimension is weighed using the parameter $\mu$. 


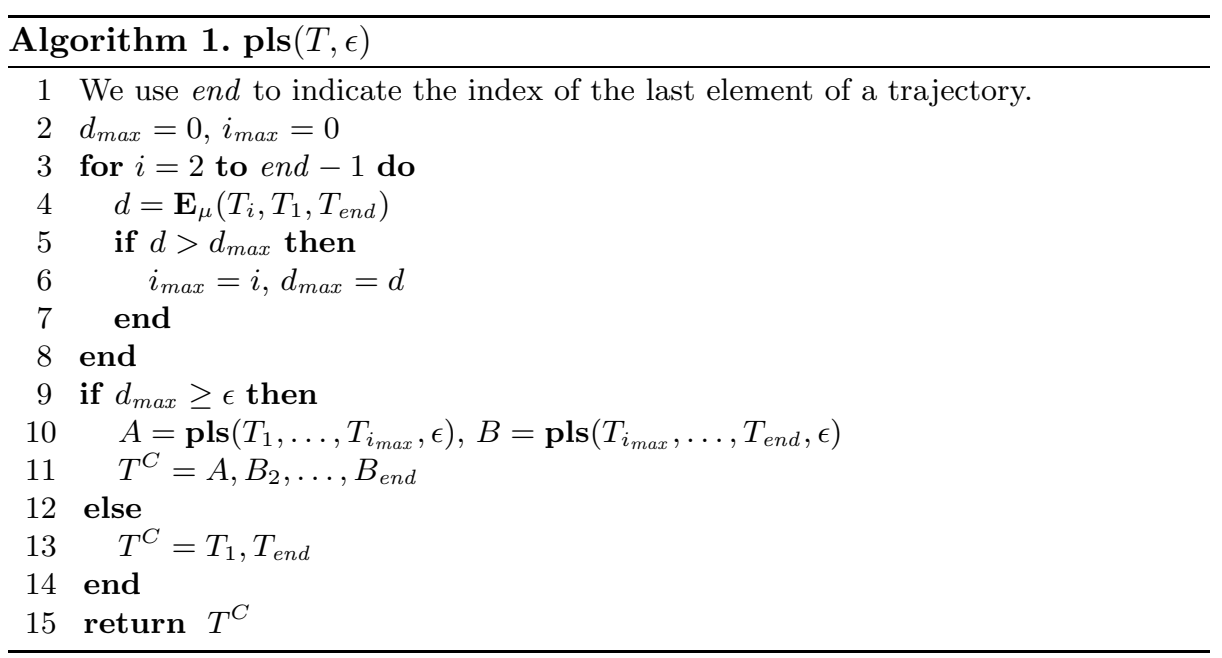

\section{Definition 14}

$$
\mathbf{E}_{\mu}\left(\left\langle x_{i}, y_{i}, t_{i}\right\rangle,\left\langle x_{1}, y_{1}, t_{1}\right\rangle,\left\langle x_{n}, y_{n}, t_{n}\right\rangle\right)=\left\|\left\langle x_{i}-x_{i}^{\prime}, y_{i}-y_{i}^{\prime}, \mu\left(t_{i}-t_{i}^{\prime}\right)\right\rangle\right\|,
$$

where $\left\langle x_{i}^{\prime}, y_{i}^{\prime}, t_{i}^{\prime}\right\rangle$ is the closest point on the line-segment $\left\langle x_{1}, y_{1}, t_{1}\right\rangle,\left\langle x_{n}, y_{n}, t_{n}\right\rangle$ in terms of the Euclidean distance.

\section{Evaluation}

In this section we will present a number of experiments to test the performance of the different combinations of alignment measures and kernels on the task of clustering a set of vessel trajectories. We especially investigate the effect of applying a compression algorithm to these trajectories as a preprocessing step.

\subsection{Dataset}

Our experimental dataset consist of 716 vessel trajectories originally gathered using the Automatic Identification System (AIS). A domain expert has partitioned these trajectories into 8 different clusters, creating a gold standard $G=g_{1}, \ldots, g_{8}$. The clusters are very different in size, ranging from 8 to 348 . The average length of a trajectory is a sequence of $300\langle x, y, t\rangle$ vectors. Trajectories are delimited either by the vessel leaving the area of observation or the vessel stopping. For all trajectories $t_{1}$ is set to 0 . We illustrate the clustering in Fig. 1 The general direction of movement for each of the 8 clusters is indicated with an arrow. Note that we only plotted the $x$ and $y$ dimensions and not $t$. The position data of the vessels was originally collected in a latitude, longitude format but has been converted, using a suitable map projection, to allow for normal Euclidean geometry. 


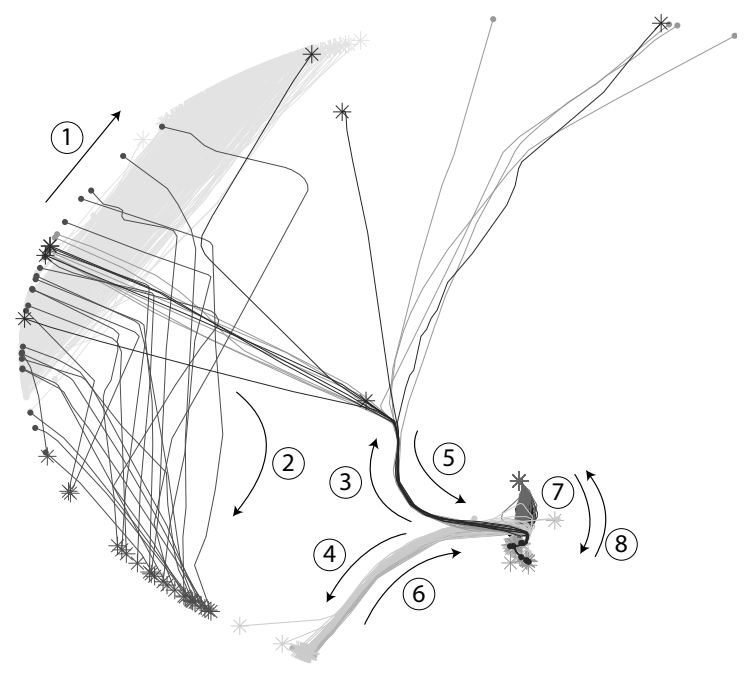

Fig. 1. An overview of the vessel trajectory dataset with the 8 different clusters of the gold standard indicated with shades of gray and an arrow giving the general direction. A solid dot indicates the start of a trajectory and an asterix indicates the end.

\subsection{Experimental Setup}

As test datasets we constructed 4 random subsets from the 716 vessels set. From each cluster in the gold standard we randomly extracted up to 20 trajectories. Some clusters contained fewer elements in which case we took them all. This resulted in 4 datasets of 138 trajectories. We did this to allow for reasonable computation times for the experiments.

Because it is unclear how to weigh the time dimension against the space dimensions, we have defined a number of settings for this. In the first setting we treated the trajectories as if they were sampled with a fixed sample rate. This means that some positions had to be linearly interpolated between two positions from the original data. In this setting a trajectory is a sequence of $\langle x, y\rangle$ points and time is implicitly represented by the fixed time interval between these points. We introduce this condition, because DTW is commonly applied to fixed sample rate time-series. Furthermore, by decreasing the sample rate we get a baseline form of compression to compare to Algorithm 1. In the other settings we tested 4 weights $w$ for the time dimension. So that a trajectory essentially becomes: $T=\left\langle x_{1}, y_{1}, w t_{1}\right\rangle, \ldots,\left\langle x_{n}, y_{n}, w t_{n}\right\rangle$. As weights we took $w=0, \frac{1}{3}, \frac{2}{3}, 1$. With $w=0$ we ignore the time dimension and with the other weights we increasingly weigh the time dimension more heavily. The $w=1$ setting means that the average difference between two points in the space dimension is roughly equal to the average difference in the time dimension 3

\footnotetext{
${ }^{3}$ To enhance readability we do not give the actual values for the weights, because they depend on the units that the dimensions are in.
} 
To investigate the influence of trajectory compression on the clustering performance we used 4 compression settings. The first setting being $\epsilon=0$, thus, we apply no compression. In the other settings we applied Algorithm 1 to each trajectory with $\epsilon=25,50,100 \mathrm{~m}$, respectively. We set the $\mu$ parameter to a value that we determined earlier and did not vary it for these experiments, however, it is a potential parameter to tune. In the fixed sample rate setting we set the sample rate to be (very) roughly equal to the compression rate achieved under the different compression settings, see Table 11 We set the sample rate for the no compression case to the average temporal distance between two consecutive trajectory samples in the uncompressed dataset, again see Table 1 .

Table 1. Average compression rate and corresponding sample rate for different $\epsilon$ settings

\begin{tabular}{lllll}
\hline & \multicolumn{4}{c}{ No compression $\epsilon=25 \mathrm{~m} \epsilon=50 \mathrm{~m} \epsilon=100 \mathrm{~m}$} \\
\hline Compression rate $(\%)$ & 0 & 96 & 97 & 98 \\
sample rate $(\mathrm{Hz})$ & 0.1 & 0.01 & 0.002 & 0.001 \\
\hline
\end{tabular}

For each of the different combinations of settings, i.e. for each combination of subset, time setting and compression setting, we computed a number of kernels. Two for the SSA measure: $K_{\max }^{\mathrm{SSA}}$ and $K_{\mathrm{sum}}^{\mathrm{SSA}}$. Two for the DTW measure: $K_{\max }^{\mathrm{DTW}}$ and $K_{\mathrm{sum}}^{\mathrm{DTW}}$. And 10 for the Edit measure; five times $K_{\max }^{\mathrm{ED}}$ and five times $K_{\mathrm{sum}}^{\mathrm{ED}}$, with $g=-0.01,-0.025,-0.05,-0.075,-0.1$.

We used each of these kernels as input for the kernel k-means clustering algorithm. The kernel k-means algorithm is run 100 times with random initializations and we keep the clustering with minimal intra cluster spread. This process is repeated 10 times. We set $k=8$, i.e. the same amount of clusters as in the gold standard. Thus for each kernel we get 10 clusterings that we evaluate against the gold standard $G$ according to the scoring function in Definition [15, taken from [14. The intuition behind this definition is that we take the best F1-score 4 for each cluster $g_{i}$ in the gold standard $G$ and average over these scores.

Definition 15. The score of a clustering $C$ of size $k$ with respect to a gold standard $G$ of size $k$ is:

$$
\operatorname{score}(C, G)=\frac{1}{k} \sum_{i=1}^{k} \max _{1 \leq j \leq k} \frac{2\left|g_{i} \cap c_{j}\right|}{\left|g_{i}\right|+\left|c_{j}\right|} .
$$

\subsection{Results}

In the results below the mean of a kernel is computed over the scores for the 4 subsets with 10 repeats each, thus for each kernel $N=40$. Per kernel we statistically compare the scores for the high compression/low sample rates settings

\footnotetext{
${ }^{4}$ The F1-score is the harmonic mean between precision and recall.
} 
to the scores for the no compression/high sample rate setting using a two-tailed student t-test with $p<0.05$.

We do not give all the mean scores because of space constraints. Also, discussion of the presented results is postponed to Sect. 5. First we will look at the performance of the different kernels under trajectory compression. Therefore we look at the time setting for which the highest scores are achieved. In Table 2 we give the results for the 14 kernels under the time setting $w=0$. Scores in bold indicate a significant difference between that clustering score, according to the above defined test, and the clustering score for the no compression case, with the bold score being higher. Scores in italic indicate a significant difference between that score and the score for the no compression case, with the score in italic being lower. For completeness we give the mean score of 40 random clusterings, which is 0.24 .

The best performing kernels for our clustering task are the $K_{\max }^{\mathrm{ED}}$ kernels. With the right $g$ setting they can achieve a perfect clustering, e.g. a score of 1.05 These kernels perform better on the compressed trajectories than on the uncompressed trajectories. However, the $K_{\text {sum }}^{\mathrm{ED}}$ kernels perform worse on the compressed trajectories. The $K_{\max }^{\mathrm{DTW}}$ kernel also performs better on the compressed data. However, it does not perform as much better on the compressed data as the $K_{\max }^{\mathrm{ED}}$ kernels. Both shortest sequence alignment kernels perform a lot worse than the other kernels. The $K_{\text {sum }}^{\mathrm{ED}}$ and $K_{\text {sum }}^{\mathrm{DTW}}$ kernels perform almost identical in the uncompressed case.

Table 2. Value of score for 14 different kernels under time setting $w=0$

\begin{tabular}{lllll}
\hline Kernel & \multicolumn{4}{c}{ No compression $\epsilon=25 \mathrm{~m} \epsilon=50 \mathrm{~m} \epsilon=100 \mathrm{~m}$} \\
\hline max, SSA & 0.52 & 0.34 & 0.35 & 0.42 \\
max, DTW & 0.87 & $\mathbf{0 . 9 0}$ & $\mathbf{0 . 9 1}$ & $\mathbf{0 . 9 1}$ \\
$\max , \mathrm{ED}, g=-0.01$ & 0.91 & 0.92 & 0.93 & $\mathbf{0 . 9 4}$ \\
$\max , \mathrm{ED}, g=-0.025$ & 0.88 & $\mathbf{1 . 0}$ & $\mathbf{1 . 0}$ & $\mathbf{1 . 0}$ \\
$\max , \mathrm{ED}, g=-0.05$ & 0.88 & $\mathbf{1 . 0}$ & $\mathbf{1 . 0}$ & $\mathbf{1 . 0}$ \\
$\max , \mathrm{ED}, g=-0.075$ & 0.88 & $\mathbf{0 . 9 3}$ & $\mathbf{0 . 9 7}$ & $\mathbf{0 . 9 9}$ \\
$\max , \mathrm{ED}, g=-0.1$ & 0.87 & $\mathbf{0 . 9 0}$ & $\mathbf{0 . 8 9}$ & $\mathbf{0 . 9 6}$ \\
sum, SSA & 0.52 & 0.34 & 0.35 & 0.33 \\
sum, DTW & 0.87 & 0.82 & 0.85 & 0.87 \\
sum, ED, $g=-0.01$ & 0.87 & 0.62 & 0.63 & 0.65 \\
sum, ED, $g=-0.025$ & 0.87 & 0.64 & 0.64 & 0.63 \\
sum, ED, $g=-0.05$ & 0.87 & 0.63 & 0.63 & 0.66 \\
sum, ED, $g=-0.075$ & 0.87 & 0.64 & 0.63 & 0.66 \\
sum, ED, $g=-0.1$ & 0.87 & 0.64 & 0.63 & 0.67 \\
\hline
\end{tabular}

Next we will look at the effect of the different time settings. In Table 3 we give a selection of kernel results for the fixed sample rate time setting. We have included

${ }^{5}$ This is the rounded average of all 40 runs, not all runs get a score of 1.0. 
the two edit distance kernels with the best performance and also added the best performing kerne $6\left(K_{\max , g=-0.05}^{\mathrm{ED}}\right)$ for the $w=0$ setting. Bold and italic have a similar meaning as in the previous table. Furthermore, ${ }^{+}$indicates a significant difference with the corresponding value from Table 2 if this difference is positive. This is similar for ${ }^{-}$but the difference is negative. We can see that there are better performing kernels in the $w=0$ setting. Table 3 also shows that low frequency fixed sample rates $(\leq 0.01 \mathrm{~Hz})$ do not degrade the performance of a number of kernels and sometimes even improve it.

Table 3. Value of score for a selection of 7 different kernels under the fixed sample rate setting

\begin{tabular}{lllll}
\hline Kernel & $0.1 \mathrm{~Hz}$ & $0.01 \mathrm{~Hz}$ & $0.002 \mathrm{~Hz}$ & $0.001 \mathrm{~Hz}$ \\
\hline max, SSA & $0.47^{-}$ & $0.46^{+}$ & $0.47^{+}$ & $0.45^{+}$ \\
$\max , \mathrm{DTW}$ & 0.87 & $0.87^{-}$ & $0.87^{-}$ & $0.87^{-}$ \\
max, ED, $g=-0.01$ & $0.95^{+}$ & 0.94 & $\mathbf{0 . 9 7 ^ { + }}$ & 0.94 \\
max, ED, $g=-0.05$ & $0.87^{-}$ & $\mathbf{0 . 8 7 ^ { - }}$ & $\mathbf{0 . 8 7 ^ { - }}$ & $0.87^{-}$ \\
sum, SSA & $0.48^{-}$ & $0.39^{-}$ & $0.29^{-}$ & $0.26^{-}$ \\
sum, DTW & $0.86^{-}$ & $0.71^{-}$ & $0.70^{-}$ & $0.75^{-}$ \\
sum, ED, $g=-0.01$ & $0.86^{-}$ & 0.62 & $0.61^{-}$ & $0.59^{-}$ \\
\hline
\end{tabular}

In Table 4 we give the results for a selection of kernels for the setting $w=\frac{1}{3}$. We have taken the same edit distance kernels as in Table 3, which also includes the best performing one for this setting. Again, ${ }^{+}$and ${ }^{-}$indicate statistical significance compared to the same kernel for the $w=0$ setting. We see that there are better performing kernels in the $w=0$ setting.

Table 4. Value of score for a selection of 7 different kernels under time setting $w=\frac{1}{3}$

\begin{tabular}{lllll}
\hline Kernel & \multicolumn{4}{l}{ No compression $\epsilon=25 \mathrm{~m} \epsilon=50 \mathrm{~m} \epsilon=100 \mathrm{~m}$} \\
\hline max, SSA & 0.52 & $0.36^{+}$ & $0.42^{+}$ & $0.52^{+}$ \\
max, DTW & $0.87^{-}$ & $\mathbf{0 . 8 9}^{-}$ & $\mathbf{0 . 8 9}$ & $\mathbf{0 . 9 0}$ \\
max, ED, $g=-0.01$ & $0.89^{-}$ & $0.82^{-}$ & $0.80^{-}$ & $0.80^{-}$ \\
max, ED, $g=-0.05$ & 0.88 & $\mathbf{0 . 9 5}^{-}$ & $\mathbf{0 . 9 8}$ & $\mathbf{0 . 9 9}$ \\
sum, SSA & 0.51 & 0.33 & 0.35 & 0.33 \\
sum, DTW & $0.83^{-}$ & 0.82 & 0.84 & $\mathbf{0 . 8 6}$ \\
sum, ED, $g=-0.01$ & $0.83^{-}$ & 0.63 & 0.64 & 0.65 \\
\hline
\end{tabular}

The performance for the time settings $w=\frac{2}{3}, 1$ is worse than the results for $w=\frac{1}{3}$. Thus, we did not consider it necessary to include them. We furthermore remark that the difference in mean score between the best performing kernel in

${ }^{6}$ Based on the non-rounded scores. 
the $w=0$ setting $\left(K_{\max , g=-0.05}^{\mathrm{ED}}, \epsilon=50 \mathrm{~m}\right.$, score $\left.=1.0\right)$ and the best performing kernel in the fixed sample rate setting $\left(K_{\max , g=-0.01}^{\mathrm{ED}}, 10 \mathrm{~Hz}\right.$, score $\left.=0.95\right)$ is significant.

We give some results on computation time in Table 5. These computation times are for running our MatLab implementation of the DTW alignment kernels 7 on an AMD Phenom II X4 955 (3.2 Ghz) cpu system with 4 GB of ram. The computation time for a kernel on the full dataset would be around a factor 40 larger. This does not make it intractable, but did not allow us enough flexibility in performing experiments.

Table 5. Running time for computing one DTW kernel. In the compression cases, the computation time needed for compression is included.

\begin{tabular}{lllll}
\hline \multicolumn{4}{l}{ Kernel type No compression $\epsilon=25 \mathrm{~m} \epsilon=50 \mathrm{~m} \epsilon=100 \mathrm{~m}$} \\
\hline $\max$ & $330 \mathrm{~s}$ & $2.4 \mathrm{~s}$ & $1.9 \mathrm{~s}$ & $1.7 \mathrm{~s}$ \\
$\mathrm{sum}$ & $356 \mathrm{~s}$ & $2.6 \mathrm{~s}$ & $2.1 \mathrm{~s}$ & $1.8 \mathrm{~s}$ \\
\hline
\end{tabular}

\subsection{Performance on the Full Dataset}

We have also computed a set of kernels on the full dataset. Because of long computation time we did not do this for the no compression/high sample rate setting. We used the same kernel k-means settings as before, which results in 10 clusterings per kernel. In general we see the same performance differences as above, but the absolute numbers are lower. The highest performance, 0.86 , is achieved by $K_{\max }^{\mathrm{ED}}$ with $g=-0.075$ under the $w=\frac{2}{3}$ and $\epsilon=50 \mathrm{~m}$ settings. However, this score does not differ significantly from the best score, 0.85 , in the $w=0$ setting, which is for $K_{\max }^{\mathrm{ED}}$ with $g=-0.025$ and $\epsilon=25 \mathrm{~m}$.

\section{Conclusions and Future Work}

The main result is that the $K_{\max }^{\mathrm{ED}}$ and $K_{\max }^{\mathrm{DTW}}$ kernels perform better on the compressed trajectories, even though we see from Tables 1 and 5 that the dataset is reduced by $96 \%$ or more, and kernel computation time is at least 100 times faster. The trajectory compression algorithm works by recursively retaining the most salient elements of a trajectory, reducing noise. Removing so many points from the trajectories can have a negative influence on the alignments. However, in our dataset, similar trajectories have similar salient elements, vessels stop and turn in the same places due to geographical and legislative constraints. This means that similar compressed trajectories can be aligned well. In such a setting applying trajectory compression as a preprocessing step can reduce computational costs significantly and, at the same time, improve performance.

7 The results are comparable for other kernels. 
That the $K_{\max }^{\mathrm{ED}}$ kernels perform better than the $K_{\max }^{\mathrm{DTW}}$ kernels on the compressed trajectories can be explained by how gaps are treated. In the edit distance case only a relatively small gap penalty is added for unaligned points. However, in the DTW case, all points have to be aligned. Because of compression, consecutive points in a trajectory can be very far apart, and repeating an element can lead to a high penalty. For the $K_{\text {sum }}^{\mathrm{ED}}$ kernels the relatively small gap penalty actually seems to work against them, since bad alignments still have a relatively high score: the worst score is a summing of just gap penalties. These high scores are a relatively big, but meaningless, part of the sum. This does not happen in the $K_{\text {sum }}^{\mathrm{DTW}}$ case, bad alignments do not get such a relatively high score. So, we see that the $K_{\text {sum }}^{\mathrm{DTW}}$ kernels perform almost as well as $K_{\max }^{\mathrm{DTW}}$ versions. The almost identical performance of the $K_{\text {sum }}^{\mathrm{ED}}$ and $K_{\text {sum }}^{\mathrm{DTW}}$ kernels in the uncompressed case is because the amount of points in a trajectory is so high that there are very little gaps compared to aligned elements, and thus the influence of how the DTW and edit kernels treat gaps is very small.

The main reason for shortest sequence alignment kernels to perform so much worse is that a trajectory of a small number of elements can still be very similar to a trajectory with a lot more elements, because we do not penalize gaps. In most cases, such trajectories are actually not very similar.

Inspection of the dataset shows that the best performance being in the $w=0$ setting is not so strange. Within one cluster most trajectories actually have similar speeds, and time is thus not so important, the sequence of the $\langle x, y\rangle$ points is enough. We have not investigated what happens if we do not set each start time of the trajectory to 0 , but, for instance, temporally align them differently, this is something for future work.

In future work we would also like to look at other types of movement data to test the influence of trajectory compression. Especially data with less structure than vessel trajectories and a larger role of the temporal component. Furthermore, it would be interesting to look at other types of clustering methods with these alignments, for instance density based ones. Finally, we would like to look at similarity measures from computational geometry, as for instance in [315].

\section{Acknowledgments}

This work has been carried out as a part of the Poseidon project in collaboration with Thales Nederland, under the responsibilities of the Embedded Systems Institute (ESI). This project is partially supported by the Dutch Ministry of Economic Affairs under the BSIK program.

\section{References}

1. Andrienko, G., Andrienko, N., Wrobel, S.: Visual analytics tools for analysis of movement data. SIGKDD Explor. Newsl. 9(2), 38-46 (2007)

2. Ben-Hur, A., Horn, D., Siegelmann, H.T., Vapnik, V.: Support vector clustering. Journal of Machine Learning Research 2, 125-137 (2001) 
3. Buchin, K., Buchin, M., van Kreveld, M.J., Luo, J.: Finding long and similar parts of trajectories. In: Agrawal, D., Aref, W.G., Lu, C.T., Mokbel, M.F., Scheuermann, P., Shahabi, C., Wolfson, O. (eds.) GIS, pp. 296-305. ACM, New York (2009)

4. Chen, L., Ng, R.: On the marriage of lp-norms and edit distance. In: VLDB 2004: Proceedings of the Thirtieth international conference on Very large data bases, pp. 792-803. VLDB Endowment (2004)

5. Cuturi, M., Vert, J.P., Birkenes, O., Matsui, T.: A kernel for time series based on global alignments. In: IEEE International Conference on Acoustics, Speech and Signal Processing, ICASSP 2007, vol. 2, pp. II-413-II-416 (2007)

6. Dhillon, I.S., Guan, Y., Kulis, B.: Weighted graph cuts without eigenvectors - a multilevel approach. IEEE Transactions on Pattern Analysis and Machine Intelligence 29, 1944-1957 (2007)

7. Douglas, D.H., Peucker, T.K.: Algorithms for the reduction of the number of points required to represent a digitized line or its caricature. Cartographica 10(2), 112-122 (1973)

8. Gaffney, S., Smyth, P.: Trajectory clustering with mixtures of regression models. In: Knowledge Discovery and Data Mining, pp. 63-72 (1999)

9. Gruber, C., Gruber, T., Sick, B.: Online signature verification with new time series kernels for support vector machines. In: Zhang, D., Jain, A.K. (eds.) ICB 2005. LNCS, vol. 3832, pp. 500-508. Springer, Heidelberg (2005)

10. Gudmundsson, J., Katajainen, J., Merrick, D., Ong, C., Wolle, T.: Compressing spatio-temporal trajectories. Computational Geometry 42(9), 825-841 (2009)

11. Gudmundsson, S., Runarsson, T.P., Sigurdsson, S.: Support vector machines and dynamic time warping for time series. In: IJCNN, pp. 2772-2776. IEEE, Los Alami$\operatorname{tos}(2008)$

12. Joder, C., Essid, S., Richard, G.: Alignment kernels for audio classification with application to music instrument recognition. In: European Signal Processing Conference (EUSIPCO), Lausanne, Suisse (2008)

13. Lee, J.G., Han, J., Whang, K.Y.: Trajectory clustering: a partition-and-group framework. In: SIGMOD 2007: Proceedings of the 2007 ACM SIGMOD international conference on Management of data, pp. 593-604. ACM Press, New York (2007)

14. Liao, T.W.: Clustering of time series data - a survey. Pattern Recognition 38(11), 1857-1874 (2005)

15. Nanni, M., Pedreschi, D.: Time-focused clustering of trajectories of moving objects. J. Intell. Inf. Syst. 27(3), 267-289 (2006)

16. Pelekis, N., Kopanakis, I., Kotsifakos, E., Frentzos, E., Theodoridis, Y.: Clustering trajectories of moving objects in an uncertain world. In: ICDM 2009: Proceedings of the 2009 Ninth IEEE International Conference on Data Mining. pp. 417-427. IEEE Computer Society, Washington (2009)

17. Shawe-Taylor, J., Cristianini, N.: Kernel Methods for Pattern Analysis. Cambridge University Press, New York (2004)

18. Vert, J.P., Saigo, H., Akutsu, T.: Local alignment kernels for biological sequences. In: Scholkopf, B., Tsuda, K., Vert, J.P. (eds.) Kernel Methods in Computational Biology, pp. 131-154. The MIT Press, Cambridge (2004)

19. Vlachos, M., Gunopoulos, D., Kollios, G.: Discovering similar multidimensional trajectories. In: ICDE 2002: Proceedings of the 18th International Conference on Data Engineering, Washington, DC, USA, p. 673. IEEE Computer Society Press, Los Alamitos (2002) 